Reis, J., Schambra, H. M., Cohen, L. G., Buch, E. R., Fritsch, B., Zarahn, E., \& Krakauer, J. W. (2009). Noninvasive cortical stimulation enhances motor skill acquisition over multiple days through an effect on consolidation. Proceedings of the National Academy of Sciences of the United States of America, 106(5), 1590-1595.

Rosenkranz, K., Nitsche, M. A, Tergau, F., \& Paulus, W. (2000). Diminution of training-induced transient motor cortex plasticity by weak transcranial direct current stimulation in the human. Neuroscience Letters, 296(1), 61-63.

Schmidt, R., \& Lee, T. (2011). Motor control and learning: A behavioral emphasis (5a Ed.). Champaign, IL: Human Kinetics.

Stagg, C. J., \& Nitsche, M. A. (2011). Physiological basis of transcranial direct current stimulation. The Neuroscientist: A Review Journal Bringing Neurobiology, Neurology and Psychiatry, 17(1), 37-53. doi:10.1177/1073858410386614.

Taylor, J. L., Butler, J. E., Allen, G. M., \& Gandevia, S. C. (1996). Changes in motor cortical excitability during human muscle fatigue. Journat of Phusiology $490(2)$

Teixeira, L. A., Chaves, C. E. O., Silva, M. V. M., \& CarvaIho, M. A. (1998). Assimetrias laterais no desempenho de habilidades motoras. Kinesis, 20, 77-92.

Todor, J., \& Smyle, A. (1985). Manual asymmetries in control motor. In E. A. Roy (Ed.), Neuropsychological studies of apraxia and related disorders. Amsterdam: North Holland.

Vines, B. W., Cerruti, C., \& Schlaug, G. (2008). Dual-hemisphere tDCS facilitates greater improvements for healthy subjects non-dominant hand compared to uni-hemisphere stimulation. BMC Neuroscience, 9, 103. doi:10.1186/1471-2202-9-103.

Vines, B. W., Nair, D. G., \& Schlaug, G. (2006). Contralateral and ipsilateral motor e efects after transcranial direct current stimulation. Cognitive Neuroscience and Neuropsychology, 17(6), 671-674.

Vitor-Costa, M., Pereira, L. A., Montenegro, R. A., Okano, A. H., \& Altimari, L. R. (2012). A estimulação transcraniana por corrente contínua como recurso ergogênico: Uma nova perspectiva no meio esportivo. Revista da Educacao Fisica, 23(2), 167-174. doi:10.4025/reveducfis.v23i2.10670.

Zimerman, M., Heise, K. F., Hoppe, J., Cohen, L. G., Gerloff, C., \& Hummel, F. C. (2012). Modulation of training by single-session transcranial direct current stimulation to the intact motor cortex enhances motor skill acquisition of the paretic hand. Stroke: $A$ Journal of Cerebral Circulation, 43(8), 2185-2191. doi:10.1161/111.645382.
AUTORES:

Margareth Regina G V de Faria

Daniela Sacramento Zanin

Grassyara Pinho Tolentino

Iransé Oliveira Silva

Patrícia Espíndola M Venâncio

https://doi.org/10.5628/rpcd.17.S3A.25

Tradução e dados preliminares

do instrumento de vitimização

para adolescentes e adultos

brasileiros a partir do Juvenile

Victimization Questionnaire

PALAVRAS CHAVE:

Violência. Psicometria.

Saúde. Adolescente.

\title{
RESUMO
}

Este artigo pretendeu descrever o processo de construção e validação da versão brasileira do instrumento de avaliação de vitimizações em adolescentes, baseada no Juvenile Questionnaire Victimization (JVQ) de Finkelhor et al. (2005). A primeira etapa de validação do JVQ foi constituída pela tradução dos itens para o português (translation). Foi realizada a tradução dos 34 itens do instrumento por dois psicólogos. Posteriormente, um grupo de juízes especialistas verificaram a compreensão dos itens e, então, foram retraduzidos para o inglês (backtranslation). Quatro adolescentes avaliaram eventuais dificuldades na compreensão dos itens, não tendo evidenciado dificuldades em compreenderem as perguntas e respondê-las objetivamente (Estudo 1). No estudo piloto (Estudo 2) participaram 132 universitários entre o 1으 e o 6으 período de psicologia. Os resultados mostraram maior relato de vitimizações testemunhadas $(n=268)$, seguidas de vitimizações por crimes convencionais $(n=213)$, vitimizações entre pares $(n=144)$ e, por último, vitimizações sexual ( $n=114)$ e maus-tratos infantis $(n=98)$. Tanto os grupos de adolescentes quanto os de universitários apresentaram boa compreensão dos itens. Dessa forma, garantiu-se que as respostas aos mesmos fossem confiáveis. Após estes procedimentos, conclui-se que o instrumento possui condições satisfatórias de validade para ser usado em novas pesquisas. 
Translation and preliminaries data of instrument victimization for Brazilian teens and adults

\section{from juvenile victimization}

\section{ABSTRACT}

This article aims to describe the process of construction and validation of the Brazilian version of victimization assessment instrument for adolescents, based on the Juvenile Victimization Questionnaire (JVQ) of Finkelhor et al. (2005). The first JVQ validation stage was the translation of the 34 items of the instrument into Portuguese (translation). The translation was carried out by two psychologists. After the translation, a group of expert judges in studies of violence analized the comprehensibility of the items, and then they were retranslated into English (backtranslation). Four adolescents evaluated eventual difficulties in understanding the questions and answer them objectively (Study 1). In the pilot study, 132 university participants between the 1st and 6th stage of psychology, reported more witnessed victimization $(n=268)$, followed by victimization by conventional crimes $(n=213)$, peer victimization $(n=144)$ and, finally, sexual victimization $(n=114)$ and child abuse $(n=98)$. Both the group of adolescents and the university students showed a good understanding of the items. Thus, it was assured that the answers to them were reliable. After these procedures, we concluded that the instrument has favorable conditions of validity to be used in further research.

\section{KEY-WORDS}

Violence. Psychometrics. Health

Adolescents.

\section{INTRODUÇÃo}

Segundo Martins (2006), o sucesso de um instrumento se dá quando ele é merecedor de créditos para solução de um problema de pesquisa. Para isso, devem ser observados alguns cuidados na construção de um instrumento relacionados a validação e a precisão do mesmo. Precisão também é conhecida por confiabilidade do instrumento. Medidas confiáveis são replicáveis e consistentes. E medidas válidas são representações precisas das características que se pretende medir.

Anastasi e Urbina (2000) afirmam que a validade refere-se a aquilo que o instrumento mede, mas também o quão bem ele faz isso. Segundo Pasquali (2003), validade de um instrumento psicológico é a verificação de que ele mede o que se propõe a medir, pois "ao se medirem os comportamentos (itens), que são a representação do traço latente, está-se medindo o próprio traço latente" (p. 162). No entanto, isto só é possível se existir uma teoria prévia do traço que fundamente que tal representação comportamental constitui uma hipótese dedutível da teoria. Assim, a validade do instrumento se dá pela testagem empírica da verificação da hipótese.

Atualmente, os manuais de psicometria trazem como principais formas de validação: a validade de conteúdo, a validade de critério e a validade de constructo (Pasquali, 2003). Martins (2006) acrescenta que a validade aparente é necessária para que o instrumento não pareça tolo aos respondentes. Assim, a validade de um instrumento pode ser avaliada por algumas evidências: validade aparente, validade de conteúdo, validade de critério e validade de constructo.

Na validade aparente, avalia se a medida de fato parece medir o que se propõe, considerando a definição teórica. Pode ser realizada pela avaliação de juízes sobre os itens, considerando a população alvo (Martins, 2006).

A validade de conteúdo é o grau em que a medição representa o conceito que se pretende medir (Martins, 2006). Envolve uma avaliação do conteúdo do instrumento para verificar se ele abrange uma amostra representativa do que se pretende medir (Anastasi, 1977). A validade de conteúdo é garantida pela técnica de construção do instrumento. Para viabilizar um instrumento precisa-se seguir três especificações: (a) definição do conteúdo; (b) explicitação dos processos psicológicos (os objetivos) a serem avaliados; e (c) determinação da proporção relativa de representação no teste de cada tópico do conteúdo . Novos estudos podem aproveitar de um constructo utilizado por outros pesquisadores para garantir que todo o conteúdo do instrumento esteja sendo avaliado.

A validade de critério estabelece a validade de um instrumento comparando-o com algum critério externo (validade empírica). Se o critério se fixa no futuro, tem-se a validade preditiva, onde o instrumento prediz o comportamento futuro dos indivíduos. A validade de predizer é estabelecida através de resultados do teste com subsequente medida de um critério . Neste caso, a validade pode ser verificada comparando os resultados obtidos com outros existentes em outras pesquisas. 
A validade de constructo se refere ao grau em que um instrumento de medidas se relaciona consistentemente com outras medições assemelhadas derivadas da mesma teoria e conceitos que estão sendo medidos (Martins, 2006). Assim, a validade total será dada pela soma das validades de conteúdo, critério e constructo.

Outro aspecto importante na construção e na utilização de um instrumento de medida é quanto se utiliza uma medida desenvolvida em uma cultura e língua para outra cultura e língua. Para adaptação de um instrumento de uma cultura para a outra, devem-se seguir algumas etapas fundamentais (Borsa, Damásio, \& Bandeira, 2012). Primeiramente, traduz-se o instrumento para o novo idioma. Os autores sugerem que sejam produzidas duas versões para serem comparadas e sintetizadas. Nesse processo, um comitê (os juízes) devem avaliar as duas versões em quatro áreas: equivalência semântica (se as palavras representam o mesmo significado); equivalência idiomática (adaptação de termos difíceis para uma expressão equivalente no novo idioma); equivalência experiencial (se é aplicável à nova cultura); equivalência conceitual, se termos e expressões foram traduzidos adequadamente (Borsa et al., 2012).

Em seguida, verificando-se as versões decide-se uma síntese dos instrumentos em uma única versão. A terceira etapa é a avaliação do instrumento pelo público alvo para verificar se as instruções são claras e os termos são bem compreendidos pelo publico alvo. As sugestões do público alvo são acatadas e os termos revisados e adaptados. Nesta fase ainda não se realiza nenhum tipo de análise estatística. Na quarta etapa, procede-se para o estudo piloto para verificar se o instrumento pode realmente ser utilizado (Borsa et al., 2012).

Percebe-se que a construção e adaptação de um instrumento não é algo fácil e, talvez, por isso poucos instrumentos são completos na avaliação de um fenômeno. Em relação à violência, os estudos que tratam sobre seus vários tipos são raros. Mesmo porquê os tipos de violência se modificam conforme as etapas de desenvolvimento. Assim, por toda complexidade de construção e validação de instrumentos de avaliação, as pesquisas tendem a focar em algum tipo específico de violência. Além disso, as pesquisas costumam se concentrar nos tipos mais comuns como violência física (Reichenheim \& Moraes, (2003) ou sexual (Habigzang, Dala Corte, Hatzenberger, Stroeher, \& Koller, 2008; Mannarino, Cohen, \& Berman, 1994) deixando de lado as violências indiretas ou psicológicas, por exemplo.

No entanto, a caracterização de violência contra adolescentes de 10 a 19 anos, no Brasil, mostra que a violência psicológica, onde entrariam as vitimizações testemunhadas e indiretas, tem aumentado significativamente, ocupando o segundo lugar com 50\% dos casos, perdendo somente para violência sexual (56\%) (Ministério da Saúde do Brasil, 2008). Porém, poucos estudos são realizados com o objetivo de construção de instrumentos de avaliação para violência psicológica (Avanci, Assis, Santos, \& Oliveira, 2005).
Os resultados de pesquisas sobre instrumentos para avaliação de violência contra adolescentes (Finkelhor \& Dziuba-Leatherman, 1994; Finkelhor, Hambyb, Ormrod, \& Turner 2005; Hamby \& Finkelhor, 2004; Hamby, Finkelhor, Ormrod, \& Turner, 2004) resultaram em um instrumento que avalia cinco tipos de vitimizações em uma única aplicação, o Juvenile Questionnaire Victimization (JVQ). O JVQ contém perguntas de triagem de cerca de 34 vitimizações contra adolescentes subdivididas em cinco áreas gerais de preocupação: crimes convencionais (oito itens), maus-tratos infantis (quatro itens), violência entre pares (cinco itens), vitimização sexual (oito itens), e vitimização testemunhada ou indireta (nove itens). Cada uma dessas cinco áreas é um módulo do JVQ. Nos dois estudos apresentados neste artigo o instrumento utilizado foi o JVQ traduzido para o português e adaptado para linguagem acessível à população de adolescentes e adultos.

Nos estudos originais, a coleta de dados foi feita por entrevista via telefone por profissionais especializados neste tipo de entrevista com crianças e adolescentes. A seleção dos números foi aleatória e a seleção dos participantes se deu pela criança de aniversário mais recente. Se a criança selecionada tivesse de 2 a 8 anos a entrevista era procedida com um responsável, na maioria das vezes a mãe biológica da criança (68\%). Se a criança tivesse entre 9 e 17 anos ela mesma respondia a entrevista. As perguntas eram relativas a algum tipo de vitimização sofrida no último ano, mas os autores orientam que o instrumento também pode ser adaptado para perspectiva de vida e para relatar acontecimentos retrospectivos da infância de adultos (Finkelhor et al., 2005).

Os resultados mostraram que uma ocorrência de vitimização favorece outras ocorrências ou múltiplas vitimizações ou polivitimizacão. Em média, crianças e adolescentes pesquisados foram vitimizados de três maneiras diferentes em incidentes separados durante o período de um ano (Finkelhor et al., 2005). Os estudos de Finkelhor et al. (2005) põe em evidência a necessidade de uma avaliação conjunta de vitimizações e a compreensão dos diferentes tipos de vitimizações como relacional. Além disso, esse instrumento permite avaliar de forma mais global e diferencialmente as consequências das vivências de diferentes vitimizações e de combinações de vitimizações (revitimizações e polivitimizações) na vida das pessoas.A presente pesquisa descreve dois estudos. No estudo 1, objetiva-se: (a) descrever o processo de tradução para a versão brasileira do instrumento de avaliação de vitimizações em adolescentes e adultos a partir do JVQ. No Estudo 2 objetiva-se obter dados preliminares por meio de um estudo piloto utilizando o instrumento de avaliação de vitimização de adolescentes e adultos do Estudo 1. 


\section{ESTUDO}

\section{TRADUÇÃO DO INSTRUMENTO}

\section{MÉTODO}

PARTICIPANTES

Participaram da tradução do JVQ três juízes especialistas no tema, dois doutores e um mestre.

PROCEDIMENTO

Foi realizada a tradução do instrumento para o português e, em seguida, feitas as adaptações para uma linguagem acessível aos participantes. Posteriormente, um juiz especialista na área e com domínio da língua inglesa realizou a backtranslation.

\section{RESULTADOS}

No processo de adaptação e validação para o português, o JVQ passou pela etapa de análise teórica dos itens (Pasquali, 2003), onde se verifica a compreensão das tarefas, também chamada de análise semântica; e análise de juízes, onde se avalia a pertinência dos itens. 0 objetivo desta etapa foi de verificar a compreensão da linguagem das perguntas após tradução para o português.A primeira etapa de validação do JVQ foi constituída pela tradução dos itens para o português (translation). Foi realizada a tradução dos 34 itens do instrumento por dois psicólogos. Após a tradução, um grupo de juízes especialistas em estudos sobre violência verificaram a compreensão dos itens e retradução para o inglês (backtranslation). Após a retradução os itens foram comparados entre si e reformulados quando não correspondiam de todo à tradução.

Todos os itens do instrumento original foram mantidos, pois os juízes entenderam que todos eram pertinentes na avaliação de vitimização contra adolescentes.

\section{AVALIAÇÃO DA TRADUÇÃO DO INSTRUMENTO}

\section{MÉTODO}

PARTICIPANTES

Participaram desta etapa de pesquisa quatro adolescentes, três do sexo masculino e um do sexo feminino. Os participantes tinham entre 12 e 13 anos.
PROCEDIMENTO

Os participantes faziam parte do Programa de Erradicação do Trabalho Infantil (PETI) de um Bairro da região leste de Goiânia, Brasil. Foram selecionadas na medida em que encontravam-se em intervalos entre as atividades do programa e após seus responsáveis terem sido informados sobre os objetivos da pesquisa e concordado com a participação de seus filhos através da assinatura do Termo de Consentimento Livre e Esclarecido (TCLE). Em seguida, iniciou-se a aplicação das entrevistas utilizando-se do instrumento pelo pesquisador. As questões do instrumento foram lidas pelo pesquisador para os participantes do estudo e as respostas foram anotadas no formulário.

\section{RESULTADOS}

Os participantes não demonstraram dificuldade na compreensão dos itens e responderam coerentemente as perguntas do questionário, sem solicitação de esclarecimentos adicionais.

Houve maior relato de vitimizações testemunhadas e indiretas (18), seguida de vitimizações por crimes convencionais (13), vitimizações entre pares (8) e, por último, vitimizações por maus tratos de crianças (4) e vitimizações sexuais (4). Analisando os resultados por sujeito, verificou-se que três dos participantes afirmaram ter sofrido vitimizações em mais de $50 \%$ dos itens do instrumento, ou seja, foram polivitimizados (QUADRO 1).

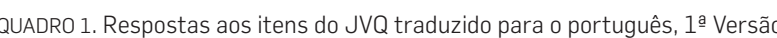

\begin{tabular}{|c|c|c|c|c|c|}
\hline & SUJEITO 1 & SUJEITO 2 & SUJEITO 3 & SUJEITO 4 & \\
\hline SEXO & FEMININO & MASCULINO & MASCULINO & MASCULINO & \\
\hline IDADE & 12 & 13 & 13 & 12 & TOTAL \\
\hline Vitimizações por Crime & 6 & 2 & 1 & 4 & 13 \\
\hline Vitimização por Maus-tratos & 1 & 1 & 2 & 0 & 4 \\
\hline Vitimizações por Pares & 3 & 2 & 3 & 0 & 8 \\
\hline Vitimização Sexual & 3 & 0 & 1 & 0 & 4 \\
\hline Vitimização Indireta & 6 & 7 & 5 & 0 & 18 \\
\hline TOTAL & 19 & 12 & 15 & 1 & \\
\hline
\end{tabular}

O objetivo deste artigo foi à tradução e validação do instrumento de vitimização para adolescentes e adultos. Verificou-se que não houve dificuldade dos adolescentes em compreender as perguntas e respondê-las objetivamente e, algumas vezes, complementando as respostas relatando os momentos em que ocorreram os eventos.

Os resultados desta primeira parte do estudo corroboram com estudos realizados anteriormente, demonstrando que em um único evento podem acontecer várias vitimizações (Finkelhor et al., 2005) e uma vitimização pode aumentar a probabilidade da ocorrência 
de outras vitimizações (Finkelhor, Ormrod, \& Turner, 2007). Após esta etapa, os itens foram submetidos à nova avaliação e transformados em afirmações para que pudessem ser aplicados em formato de escala nominal a um maior número de sujeitos no estudo piloto.

\section{ESTUDO 2}

\section{DADOS PRELIMINARES}

\section{MÉTODO}

PARTICIPANTES

Os estudantes universitários foram escolhidos para fazerem parte deste estudo piloto por tratarem de uma amostra mais acessível aos pesquisadores e por possuírem maior repertório linguístico, podendo ser mais críticos a tradução dos itens do que alunos de ensino fundamental ou médio. 0 objetivo desta etapa foi de verificar a compreensão das perguntas, após tradução para o português em um extrato mais alto da população meta, os jovens (Estatuto da Juventude, 2013) e adultos, bem como fornecer dados preliminares sobre vitimização, revitimização e polivitimização.

Participaram desta etapa 132 universitários entre o 1ำ e o 6으 período de psicologia, de três instituições privadas de uma cidade do centro oeste brasileiro. A escolha destas instituições ocorreu por acessibilidade ou conveniência.

\section{PROCEDIMENTOS}

Neste estudo piloto respeitou-se a equivalência da estrutura do instrumento. Foram mantidos os mesmos números de fatores do instrumento original (Borsa et al., 2005): vitimização por crime convencional, vitimização por maus-tratos, vitimização por pares, vitimização sexual e vitimização testemunhada ou indireta. Contudo, as perguntas foram transformadas em escala nominal, em que o participante foi solicitado a confirmar ou não a ocorrência de vitimização em cada item.

Após a exposição dos objetivos e riscos da pesquisa, todos os participantes concordaram em participar da pesquisa através da assinatura do Termo de Consentimento Livre e Esclarecido (TCLE), no qual mediante todas as aprovações o instrumento foi liberado para ser respondido.

\section{RESULTADOS}

A idade média dos participantes foi de 21.75 anos (mínimo $=17$ anos; máximo $=44$ anos e DP $=5.092)$ com predominância do sexo feminino. Dentre os estudantes, $86.3 \%$ eram solteiros e $13.7 \%$ casados ou em união estável; $33.3 \%$ eram somente estudantes e $38.1 \%$ trabalhavam mais de 6 horas por dia com vínculo empregatício (QUADRO 2).

\begin{tabular}{lccc}
\hline & $\mathrm{N}$ & $\%$ & \% ACUMULADA \\
\hline Somente Estudante & 8 & 33.3 & 33.3 \\
\hline Eventual & 1 & 1.2 & 34.5 \\
\hline Autônomo & 3 & 3.6 & 38.1 \\
\hline Trabalha até 6h por dia (com vínculo empregatício) & 13 & 15.5 & 53.6 \\
\hline Trabalha mais de 6 horas por dia (com vínculo empregatício) & 32 & 38.1 & 91.7 \\
\hline Estagiário (a) & 7 & 8.3 & 100.0 \\
\hline Total de válidos & 84 & 100.0 & \\
\hline Não respondidos & 48 & & \\
\hline TOTAL & 132 & & \\
\hline
\end{tabular}

Em relação aos resultados das vitimizações, apresentados no quadro 3, verificou-se que em primeiro lugar, houve maior relato de vitimizações testemunhadas $(n=268)$, em segundo, de vitimizações por crimes convencionais $(n=213)$, em terceiro, vitimizações por pares $(n=144)$ e, por último, vitimizações sexual $(n=114)$ e maus-tratos infantis $(n=98)$.

Avaliando por crivos, percebe-se que em crime convencional $29,7 \%$ dos sujeitos não sofreram vitimizações, mas $27.3 \%$ sofreram uma vitimização e $43 \%$ sofreram pelo menos duas vitimizações do mesmo tipo em um ano. No crivo de maus tratos, $43.4 \%$ não sofreram vitimizações, mas $39.5 \%$ tiveram pelo menos um episódio e $17 \%$ sofreram de dois a três episódios em um ano (QUADRO 3).

Na vitimização por pares, $38.9 \%$ não foram vitimizados. No entanto, $26.7 \%$ foram vitimizados uma vez e $23.7 \%$ duas vezes. Na vitimização sexual, $48.1 \%$ não foram vitimizados, $30.2 \%$ foram uma vez, $14 \%$ duas vezes e $7.8 \%$ pelo menos três vezes em um ano.

Na vitimização testemunhada os números mudam e mostram que a frequência das vitimizações aumentam, pois $25.8 \%$ nunca foram vitimizados, $15 \%$ foram uma vez, $17.5 \%$ duas vezes, $15.8 \%$ três vezes, $12.5 \%$ quatro vezes e $13.3 \%$ pelo menos cinco vezes em um ano. No geral, a média de vitimizações por sujeitos foi de $6.47(\mathrm{DP}=4.60)$, demonstrando uma alta vitimização dos participantes.

QUADRO 3. Frequências e porcentagens das vitimizações sofridas pelos participantes.

\begin{tabular}{|c|c|c|c|c|c|c|}
\hline \multicolumn{7}{|c|}{ VITIMIZAÇÕES } \\
\hline & & Crime Convencional & Maus Tratos & Por Pares & Sexual & Testemunhada \\
\hline \multirow{2}{*}{$n$} & Válidos & 128 & 129 & 131 & 129 & 120 \\
\hline & Perdas & 4 & 3 & 1 & 3 & 12 \\
\hline \multicolumn{2}{|c|}{ Média } & 1.664 & 0.756 & 1.099 & 0.884 & 2.233 \\
\hline \multicolumn{2}{|c|}{ Mediana } & 1.0 & 1.0 & 1.0 & 1.0 & 2.0 \\
\hline
\end{tabular}




\begin{tabular}{lccccc}
\hline DP & 1.64713 & 0.78834 & 1.11531 & 1.14310 & 1.94346 \\
\hline Mínimo & .00 & .00 & .00 & .00 & .00 \\
\hline Máximo & 8.00 & 3.00 & 4.00 & 5.00 & 9.00 \\
\hline Soma & 213.00 & 98.00 & 144.00 & 114.00 & 268.00 \\
\hline
\end{tabular}

\section{DISCUSSÃo}

Este estudo objetivou avaliar a compreensão da linguagem do JVQ por adolescentes e adultos e descrever dados preliminares sobre as vitimizações nesses grupo. Os resultados demonstram que as quantidades de vitimizações sexuais aumentam, comparadas com as vitimizações aos adolescentes do primeiro estudo. Apesar de não ser possível comparações, devido a amostras muitos diferentes e discrepantes em quantidade, foi um fato curioso e chamou a atenção, pois seguiu-se o mesmo padrão dos dados de vitimizações entre os adolescentes do primeiro estudo, só invertendo as posições em relação a vitimização sexual e maus tratos, no segundo. Estes resultados demonstram a ocorrência de mais de um tipo de vitimização pelo mesmo participante no período de um ano. Segundo Finkelhor et al. (2005), a ocorrência de múltiplas vitimizações pode ser denominada polivitimização. Dessa forma, uma análise mais detalhada destes dados poderia demonstrar as relações entre estas variáveis.

A compreensão dos itens foi considerada adequada e garantiu que as respostas aos mesmos fossem confiáveis. Após estes procedimentos, conclui-se que o instrumento possui condições satisfatórias de validade para ser usado em novas pesquisas.

Atendendo as sugestões dos participantes, as alterações foram feitas em todos os itens, retirando-se a expressão "no último ano" do início do item. Para o estudo definitivo, será incluído uma coluna, ao final das afirmações, onde as respostas poderão ser dadas em relação ao "último ano" e "ao longo da vida", facilitando a compreensão e lembrando sempre o participante que período da vida ele deve avaliar antes de responder. Além disso, aumentamos as possibilidades de análises e comparações, na medida em que incluímos uma nova variável comparativa para as situações apresentadas no último ano.

\section{CONCLUSÕES}

A partir deste estudo é possível contabilizar as vitimizações ocorridas nos grupos avaliados sem causar grandes constrangimentos. Além disso, é possível realizar análises comparativas entre os crivos de vitimizações após a soma dos eventos. Porém, o JVQ avalia eventos dos últimos 12 meses, não considerando alguma situação importante ocorrida em tempos anteriores. Para novos estudos sugere-se incluir uma possibilidade de resposta para eventos anteriores há um ano. Apesar disso, a utilização do JVQ na população brasi- leira permite avaliar as vitimizações, revitimizações e polivitimizações em vários grupos de adolescentes e adultos, permitindo análises com outras variáveis importantes como a saúde mental e a identificação dos tipos mais comuns de vitimizações segundo a faixa etária, permitindo a tomada de providências necessárias para a diminuição dessas ocorrências e seus consequentes prejuízos.

\section{FONTES DE FINANCIAMENTO}

Fapeg - Fundação de Amparo a Pesquisa do Estado de Goiás. 
AUTORES

Grassyara Pinho Tolentino

Ricardo Jacó de Oliveira

Patrícia Espíndola M Venâncio

Viviane Soares

Margareth Regina G V de Faria

Guilherme Nogueira de Oliveira

Claudio Battaglini

V. C. (2005). Escala de violência psicológica contra adolescentes. Revista de Saúde Publica, 39(5), 702-8.

Adaptação e validação de instrumentos psicológicos.

Paidéia, 22(53), 423-432.

Estatuto da Juventude. (2013). Lei no 12.852, de 5 de Agosto de 2013. Brasília: Senado.

Finkelhor, D., \& Dziuba-Leatherman, J. (1994). Victimization of children. American Psychological Association, 49(3), 173-183

Finkelhor, D., Hambyb, S. L., Ormrod, R., \& Turner, R. (2005). The Juvenile Victimization Questionnaire: Reliability, validity, and national norms. Child Abuse $\mathcal{E}$ Neglect, 29, 383-412

Finkelhor, D., Ormrod, R. K., \& Turner, H. A. (2007). Re-victimization patterns in a national longitudinal sample of children and youth. Child Abuse \& Neglect, 31, 479-502. Habigzang, L. F., Dala Corte, F., Hatzenberger, R., Stroeher, F. \& Koller, S. H. (2008). Avaliação psicológica em casos de abuso sexual na infância e adolescência. Psicologia: Reflexão e Crítica, 21(2), 338-344.

Hamby, S. L., \& Finkelhor. D. (2004). The Compreensive Juvenile Victimization Questionnaire. Durham, NH: ve Javenlie Victmization Question Hamby, S. L., Finkelhor, D. Ormrod, R. K., \& Turner, H. A. (2004). The Juvenile Victimization Questionnaire (JVQ): Administration and scoring manual. Durham, NH: Crimes Against Children Research Center.

Mannarino, A. P., Cohen, J. A., \& Berman, S. R. (1994). The children's attributions and perceptions scale: A new measure of sexurt abuse-related factors. Journal of Clinical Child Psychology, 23(2), 204-211.

Martins, G. A. (2006). Sobre confiabilidade e validade. Revista Brasileira de Gestão de Negócios, 8(20), 1-12. Ministério da Saúde do Brasil. (2008). Temático prevenção de violência e cultura de paz III. Brasília: Organização Pan-Americana da Saúde. Painel de Indicadores do SUS.

Pasquali, L. (2003). Psicometria: teoria dos testes no psicologia e na educação. Petrópolis, RJ: Vozes.

Reichenheim, M. E., \& Moraes, C. L. (2003). Adaptação transcultural do instrumento Parent-Child Conflict Tactics Scales (CTSPC) utilizado para identificar a violência contra a criança. Cadernos de Saúde Pública, 19(6), 1701-1712.

\section{RESUMO}

Estado de saúde e distresse psicológico em mulheres

sobreviventes ao câncer

\section{de mama tratadas no sistema}

\section{único de saúde}

\section{PALAVRAS CHAVE:}

Neoplasias da mama. Qualidade de vida. Doença crônica.

0 tratamento oncológico oferecido no sistema público de saúde brasileiro representa um negável avanço para a saúde. Contudo, são incertas as repercussões sobre o bem-estar das mulheres após este período. 0 objetivo deste estudo foi analisar o estado de saúde auto-referido e o distresse psicológico de mulheres submetidas ao tratamento antineoplasico oferecido pelo SUS até um ano após o seu termino. Foram avaliadas 22 mulheres com câncer de mama e comparadas a um grupo de mulheres sem condições crônicas $(n=22)$. Os instrumentos foram o POMS e MOS SF-36. Os domínios do SF-36 foram agrupados em duas variáveis, o componente físico e componente mental da saúde. Este questionário contempla, ainda, uma escala de transição que mede variações na saúde geral, quando comparado há um ano. Os resultados indicaram que as mulheres avaliadas apresentavam valores apropriados de distresse psicológico. Entretanto, a fadiga apresentou um efeito clinico moderado provocado pela doença (es =.50). A mensuração da transição da saúde quando comparado a um ano atrás indicou que cerca de $75 \%$ das mulheres tratadas perceberam alguma melhora na saúde ao longo de um ano. Entretanto, a saúde física $(p=0,001)$ e mental $(p=$ 0,001 ) apresentou reduções no grupo $C A$, apesar do tratamento bem-sucedido 